\title{
The usefulness of anthropometric measures
}

\author{
Francesco Landi · Rosa Liperoti · Graziano Onder
}

Published online: 2 July 2013

(c) Springer-Verlag Berlin Heidelberg 2013

Based on EWGSOP guidelines, muscle mass should be evaluated using the DEXA or bioelectrical impedance analysis (BIA) [1]. In our study, it was not possible to assess all participants using DEXA or BIA. Therefore, muscle mass was estimated using the measurement of the mid-arm muscle circumference (MAMC) [2].

Indeed, anthropometric measures are generally considered poor markers of muscle mass, and their role in the assessment of sarcopenia is debatable [3]. For example, Faisy et al. [4] suggested that BIA might be superior to MAMC in predicting malnutrition and negative outcomes among patients with chronic obstructive pulmonary disease and acute respiratory failure. However, as previously demonstrated, MAMC provides a simple measure of body composition. Moreover, muscle mass as assessed by MAMC was shown to be independently associated with mortality in elderly subjects $[5,6]$. MAMC estimates performed by expert personnel have shown excellent reliability [7].

Mid-arm muscle circumference estimation could be a practical and useful tool for the assessment of nutritional status and sarcopenia in older adults. In fact, it is simple, quick, inexpensive, noninvasive, and easy to use, valuable especially in those settings where DEXA and BIA are not available (i.e., nursing homes and long-term care settings). It is essential to recognize the relevance of the clinical assessment of sarcopenia and to reach a large and

This reply refers to the article available at doi:10.1007/s00394-013-549-z.

F. Landi $(\bowtie) \cdot$ R. Liperoti $\cdot$ G. Onder

Department of Gerontology, Catholic University of Sacred

Heart, Rome, Italy

e-mail: francesco.landi@rm.unicatt.it consolidated consensus on the recommended tools to measure body composition when DEXA and BIA are not accessible. Anthropometric measures (MAMC and/or calf circumference) could represent a simple and reliable instrument for the clinical assessment of nutritional status and sarcopenia.

\section{References}

1. Cruz-Jentoft AJ, Baeyens JP, Bauer JM et al (2010) Sarcopenia: European consensus on definition and diagnosis: report of the European Working Group on sarcopenia in older people. Age Ageing 39:412-423

2. Landi F, Liperoti R, Russo A, Giovannini S et al (2013) Association of anorexia with sarcopenia in a community-dwelling elderly population: results from the ilSIRENTE study. Eur J Nutr 52:1261-1268. doi:10.1007/s00394-012-0437-y

3. Thomas DR (2007) Loss of skeletal muscle mass in aging: examining the relationship of starvation, sarcopenia and cachexia. Clinical Nutrition 26:389-399

4. Faisy C, Rabbat A, Kouchakji B, Laaban JP (2000) Bioelectrical impedance analysis in estimating nutritional status and outcome of patients with chronic obstructive pulmonary disease and acute respiratory failure. Intensive Care Med 26:518-525

5. Wannamethee SG, Shaper AG, Lennon L, Whincup PH (2007) Decreased muscle mass and increased central adiposity are independently related to mortality in older men. Am J Clin Nutr 86:1339-1346

6. Landi F, Cruz-Jentoft AJ, Liperoti R, Russo A, Giovannini S, Tosato M, Capoluongo E, Bernabei R, Onder G (2013) Sarcopenia and mortality risk in frail older persons aged 80 years and older: results from ilSIRENTE study. Age Ageing 42:203-209

7. Landi F, Liperoti R, Russo A, Giovannini S, Tosato M, Capoluongo E, Bernabei R, Onder G (2012) Sarcopenia as a risk factor for falls in elderly individuals: results from the ilSIRENTE study. Clin Nutr 31:652-658 